\title{
A Brief Review on Processes for Cartilage Repair
}

\section{Carla Moura',a, Rita Santos-Richa $^{2, b}$, Susana Franco ${ }^{2, c}$, Cândida Malça ${ }^{3, d}$, Cristina Galhano ${ }^{4, e}$, Marta Henriques ${ }^{4, f}$, Pedro Morouço ${ }^{1, g^{*}}$}

${ }^{1}$ Centre for Rapid and Sustainable Product Development, Polytechnic Institute of Leiria, Portugal

${ }^{2}$ Sport Sciences School of Rio Maior, Polytechnic Institute of Santarém, Portugal

${ }^{3}$ Coimbra Institute of Engineering, Polytechnic Institute of Coimbra, Portugal

${ }^{4}$ College of Agriculture, Polytechnic Institute of Coimbra, Portugal

accarla.moura@ipleiria.pt, britasantosrocha@esdrm.ipsantarem.pt, 'csfranco@esdrm.ipsantarem.pt, dcandida@isec.pt, ${ }^{\mathrm{e}}$ cicgalhano@esac.pt, 'mhenriques@esac.pt, ${ }^{\mathrm{f}}$ pedro.morouco@ipleiria.pt

Keywords: osteoarthritis, joint degeneration, tissue engineering, regenerative medicine.

\begin{abstract}
The aim of the present review was to highlight some of the available processes for cartilage repair and regeneration. Considering the high impact that cartilage degeneration has in the quality of life, in an aging society, efforts to promote better treatments are crucial. The current available processes have advantages and drawbacks, that should be further investigated, aiming to obtain tailored and successful repair. Finally, some suggestions for tissue engineering strategies are presented, so that the scientific community can debate pros and cons to be investigated.
\end{abstract}

\section{Introduction}

In the last years, the world has seen an increase in the number of debilitating conditions and severe pain caused by cartilage defects. Due to accidental traumas or a disease prevalence, the scientific and clinical community are aware of the major problem that our society is facing. Osteoarthritis (OA) is a chronic condition triggered by the failure of the joints' cartilage, so that bone articulates on bone $[2,3]$. Some estimates point that this disease occurs in $9.6 \%$ of men and $18 \%$ of women [2]. This disease is on the top list concerns of WHO, being the breakdown of articular cartilage a major health matter to which there are few effective solutions [4]. Direct trauma, chronic degeneration (i.e., mechanical overload), or an abnormality of the underlying subchondral bone $[5,6]$ are the main detailed factors to produce articular cartilage lesions. In fact, regardless of the low metabolic activity and relatively poor ability to heal of chondrocytes, it is known that articular cartilage is a dynamic and responsive tissue, and the contribution of cell-produced extracellular matrix (ECM) components has a noteworthy role [6-12].

Articular cartilage (hyaline cartilage on the articular surfaces of bones) has extraordinary mechanical properties (elastic modulus of $\sim 123 \mathrm{MPa}$; mechanical tensile strength of $17 \mathrm{MPa}$; compressive modulus varying between 0.53 and $1.82 \mathrm{MPa}$; compressive stress between 14-59 MPa) [11, 13-15] and lasting durability, despite its few millimetres of thickness. Its unique structure and composition provide joints with a surface that combines low friction with high lubrication, shock absorption, and wear resistance while bearing large repetitive loads throughout a person lifetime [2, $7,8,16]$. This avascular tissue, containing no neural network or lymphatic drainage, comprises only one cell type (chondrocytes) and an abundant ECM [4, 11, 14, 17-20], which is 75\% composed by collagens, $17 \%$ of negatively charged proteoglycans (protein backbone with highly branched glycosaminoglycan side chains, made up primarily of chondroitin sulphate and hyaluronic acid), $2 \%$ of DNA and adhesion glycoproteins, and elastin $[3,16]$. The lack of blood combined with a limited proliferation potential of chondrocytes limit the intrinsic healing process, by inhibiting transport of inflammatory mediators to the defect $[4,16,21]$. There is a low concentration of chondrocytes, and they are supplied with oxygen and nutrients by a combination of diffusion and fluid flow during joint loading [14]. Although the synthesis rate of glycosaminoglycan (GAG) and collagen (primarily type II) in a developing tissue ex vivo depend on gas exchange, cells remain viable under hypoxic 
conditions, which are inherent for adult cartilage in an articular joint $[6,7,22]$. Collagen fibrils combined with GAGs provide the tensile strength, load-bearing capabilities, and resilience [3].

Damaged cartilage lead to a decrease in the production of ECM components. However, the number of chondrocytes is not influenced by its degeneration [23], being the articular cartilage injuries one serious clinical problem for orthopaedic surgeons. To categorize the magnitude of these injuries and provide a better understanding of the lesion severity, cartilage lesions are graded following the International Cartilage Repair Society (ICRS) classification (cf. Figure 1); a system with five level grades:

Grade 0 - intact cartilage;

Grade 1 - superficial lesions (fissures or cracks);

Grade 2 - fissures less than half of the cartilage thickness;

Grade 3 - fissure more than half of the cartilage thickness;

Grade 4 - fissure extended until the subchondral bone.
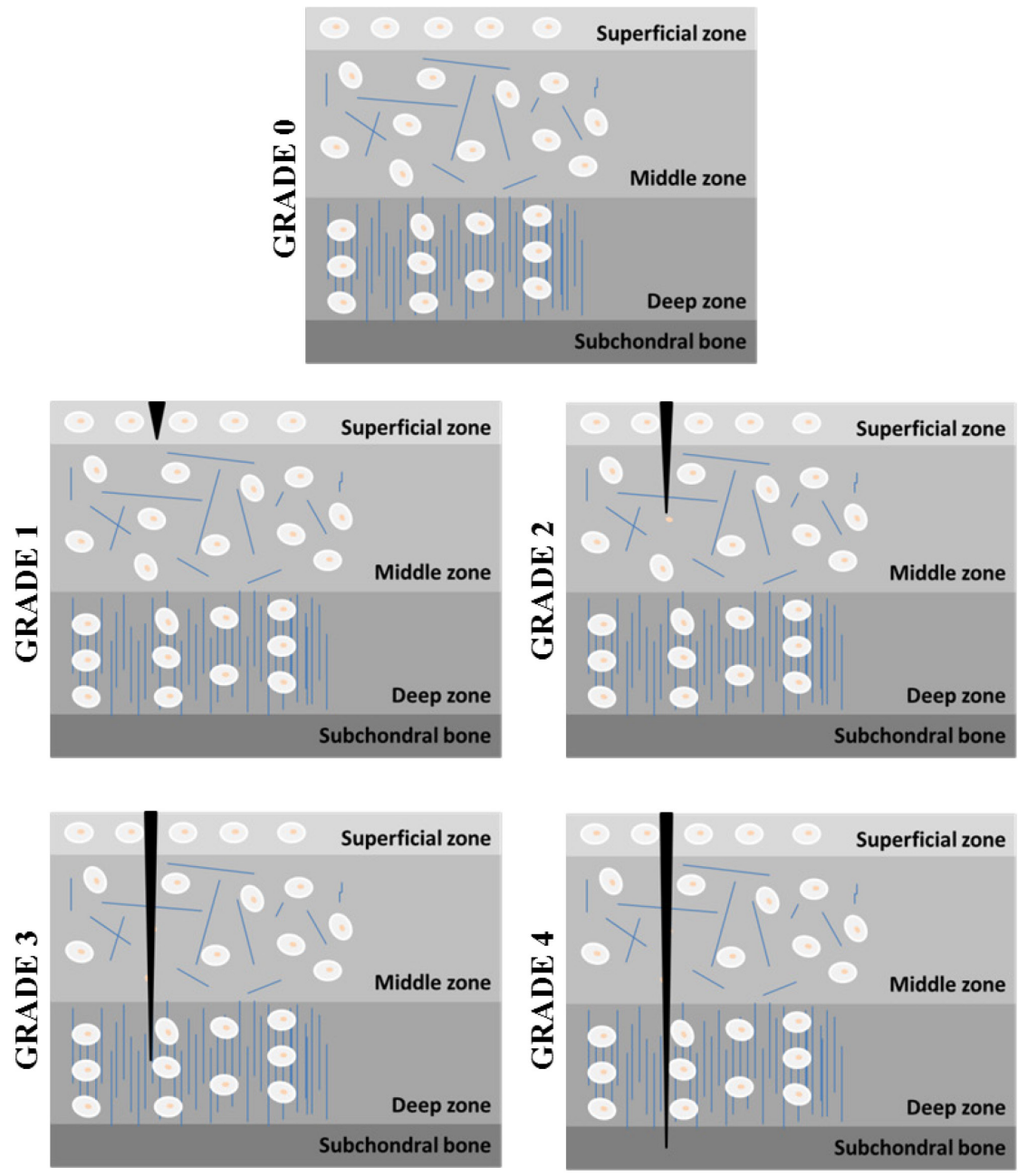

chondrocyte

Collagen

Fissure

Figure 1. Illustration of cartilage lesions according to the International Cartilage Repair Society classification.

From grade 1 to grade 3, the lesion does not reach the subchondral bone. Thus, they affect only cartilage and present a non-healing status since the blood vessels are not affected. On the other hand, in grade 4, the lesion extends to the vascularized subchondral bone. Therefore, patients will obtain some spontaneous repair once mesenchymal chondroprogenitor cells are recruited and invade the lesion to form cartilage. However, this neo-formed tissue is often fibrous and does not present the functional properties of native hyaline cartilage [24]. The relationship between cartilage stiffness and 
ICRS classification predicts a loss in stiffness of about $25 \%$ for each ICRS grade [23]. Bentley [25] classified, in the patella, defects as grade 1 with $0.5 \mathrm{~cm}$ or less in diameter, grade 2 around $1.3 \mathrm{~cm}$ or less, grade 3 with more than $1.3 \mathrm{~cm}$ and grade 4 when there is erosion down to the bone. Although there are several treatments or approaches to treating cartilage problems, which will be presented in the next section, up to the present, no generally accepted medical therapy is available for preventing cartilage loss [26].

\section{Available Clinical Treatments/Approaches for Cartilage Repair}

There are a significant number of repair strategies to treat an articular cartilage lesion; some already available, and others in an on-going research status [2, 6, 13, 14, 24, 26, 27], as follows:

- Palliative (debridement and lavage) - relief of mechanical symptoms through the elimination of cartilage fragments within the joint in cartilage-injured patients, usually older people with low mobility capacity, minimizing further delamination of the joint surface cartilage.

- Microfracturing - stimulates the formation of a fibrocartilaginous tissue through the drilling of holes of approximately $0.5-1 \mathrm{~mm}$ in diameter through the full-thickness of the articular cartilage into the bone marrow cavity to allow access to the bone marrow allows progenitor cell recruitment to the injured site.

- Grafts - chondral and osteochondral autografts (known as mosaicplasty) and allografts are used in repair full-thickness defects involving the joint. In the case of autografts cylinders are harvested from a joint area of minor load and added into pre-drilled holes at the defect site. The allografts consist in transplantation of tissue from compatible donors.

- Cell-based repair - local implantation of chondrogenic cells within a cartilage lesion for forming hyaline-like cartilage tissue. This strategy may involve the use of autologous chondrocytes or mesenchymal stem cells.

- Whole tissue transplantation - implantation of fully formed osteoarticular constructs into a defect, which can be derived from an autologous source or from an allograft donor. The use of allografts may result in some problems related with biocompatibility.

- Scaffold-based repair or cell plus scaffold-based repair - complete regeneration of articular cartilage without the inhibition of residual foreign material. Scaffolds can be used either alone or as delivery vehicles for cells, mitogens or growth factors.

- Hydrogel-based repair or cell plus hydrogel-based repair - present a high-water content and the possibility of being an injectable system.

The replacement of tissues (such as bone or cartilage) or joints with allograft materials includes the risk of infections by viruses (such as HIV, hepatitis C), graft vs. host disease [28] or even, chondrocytes can die during the process [29]. Also, the use of grafts can only be applied to damaged areas of less than $2 \mathrm{~cm}^{2}$ [24]. In addition, to contest the severe shortage of donor tissue for tissue replacement a new field emerged - Tissue Engineering - using a scaffold and/or hydrogel-based combined with cells [30].

Commercially available implants for cartilage repair (without cells)

BioCartilage ${ }^{\circledR}$ (Arthrex) is a micronized allogeneic cartilage matrix, intended to provide a scaffold for microfractures and to deliver a tissue network that can potentially signal autologous cellular interactions, thus, improving healing and quality of the tissue. It contains key components such as type II collagen, proteoglycans, and additional cartilaginous growth factors. Chondrofix ${ }^{\circledR}($ Zimmer) is an osteochondral allograft of human decellularized hyaline cartilage and cancellous bone. Multiple cylinders may be used to fill a larger defect and full recovery may take from six months to one year. DeNovo NT Graft (Zimmer) is a scaffold-free cartilage comprising manually minced cartilage tissue pieces obtained from juvenile allograft donor joints. The tissue fragments are mixed intra-operatively with fibrin glue before implantation in the prepared lesion. It is aimed to provide surgeons with an early-intervention option for the repair of different anatomic defects of articular cartilage. This graft can be applied to different articulations, such as knee, foot, ankle, elbow, shoulder or hip. Gel-One ${ }^{\circledR}$ 
Cross-linked Hyaluronate (Zimmer) is a sterile, transparent, viscoelastic hydrogel used for the treatment of pain in OA of the knee. Patients treated with this hydrogel presented great pain relief after 13 weeks of use. According to the product description available, side effects include joint swelling, joint effusion and joint pain.

\section{Cell-based commercially available implants for cartilage repair}

Autologous Chondrocyte Implantation (ACI) is another method used for cartilage repair involving the harvesting of normal chondrocytes from a minor weight-bearing area which is cultured and expanded ex vivo and then re-implanted into the defect [24]. This technique appeared in the late 1900s and was first described by Peterson et al. [14, 31]. Limitations of this procedure are related to high costs of culture conditions, graft fixation methods and lower number of cells obtained, in particular when cells must cover extensive defects, because of their proliferative limitation potential. Note that the potential for cell division (number of possible duplications) further decreases with the patient age [24]. In 1994, Brittberg et al. [32] performed ACI by injecting expanded chondrocytes into cartilage defects covered with a sutured periosteal flap, which resulted in some cases of success (11 of the 15 femoral transplants and 1 of 7 patellar transplants were successful in the appearance of hyaline cartilage). A commercially available implant based in this technique is Bio-Seed $\AA$-C (BioTissue Technologies, Freiburg, Germany) which is an autologous three-dimensional (3D) chondrocyte graft for knee cartilage defects. Cells are collected from a healthy area of the knee that is under less strain, then cells are expanded in a laboratory (approximately 3 weeks), then they are seeded in a 3D matrix. The graft provides a stable 3D environment to stimulate cells to assume their morphology and function. Homminga et al. [33] used autologous strips of perichondrium to treat the chondral defect, with fibrin glue acting as an adhesive.

Some problems related to ACI is donor site morbidity. Chondrocytes expanded in monolayer in vitro tend to suffer a process called dedifferentiation and consequently there is fibrocartilage formation at the defect site [2]. To overcome this drawback, the chondrocytes expansion is performed in the presence of transforming growth factors (TGF- $\beta 1$ or TGF- $\beta 3$ ) [24]. Despite some positive results in the formation of hyaline cartilage, the use of ACI is still questionable due to predictability and reliability of cartilage or fibrocartilage formation [12]. As a variation to ACI technique, stem cells appear as a promising cell source for cartilage repair. These cells can be adult mesenchymal stem cells (MSCs) from different origins: bone marrow (BM) [34-36], umbilical cord matrix (UCM) [37-39], adipose tissue (AT) [40, 41] or embryonic stem cells (ESCs) [42, 43]. However, there are also some limitations in the use of MSCs, such as donor variations (related to age, proliferation and differentiation potentials), uncontrolled differentiation in fibrous tissue when MSC are implanted directly, and potential immune responses when they are previously differentiated in chondrocytes. The use of ESCs is still facing challenges in purity/differentiation efficiency, homogeneity, and functionality of the cartilage tissue formation. One of the greatest challenges in this area of research is to understand, control and develop an efficient and stable culture for differentiation to a particular lineage.

\section{Tissue Engineering-implants for cartilage repair}

Cells harvested from donor tissues, including adult stem cells, can be expanded in culture and associated with resorbable biomaterials from synthetic or biological origin for implementation in the chondral defect. The advantage of incorporating materials combined with cells, is that the material matrix can provide the initial structural support required and retain cells in the defective area. This will allow ECM to be produced by cells, reconstructing the new tissue as the implanted material degrades and provide room for the new tissue [12].

A material that can be used as an implant in TE must comply a number of requirements to promote natural wound healing and tissue regeneration [44]. These materials should be biocompatible, biodegradable, processable to shapes with appropriate porosity, pore size and pore interconnectivity. Moreover, such materials should be able to support cell growth and proliferation, incorporate growth factor and present appropriate mechanical properties during all the tissue regeneration process. 
Furthermore, the development of in situ polymerizable implants that can be injected arthroscopically and function as cell delivery systems in the form of hydrogels are gaining importance in TE applications. In this case, the ideal polymer should be in liquid/viscous from, should be sterilisable without causing any chemical change, and have the capacity to incorporate biological matrix components. After its injection, it should bond to the biological surface and be cured (i.e., crosslinked) into a solid and porous structure (to allow cell adhesion, proliferation, migration, and ECM components production) with proper mechanical properties to suit the application. During the curing process, it is important to have in consideration that cells and the adjacent tissues should not be damaged [45].

Hyalograft $\AA$ C autograft is composed of cultured autologous chondrocytes seeded on a hyaluronan-based scaffold $(20 \times 20 \mathrm{~mm})$. It is used in cartilage injuries of the femoral condyle or trochlea. Chondro-gide ${ }^{\circledR}$ (Geistlich Biomaterials, Wolhusen, Switzerland) consists of a bilayer of porcine-origin collagen type I/III. It is available in three different sizes to be used by the surgeon according to the lesion: $20 \times 30,30 \times 40$ and $40 \times 50 \mathrm{~mm}$. It involves surgical procedures, for the induction of the native collagen, which will serve as a scaffold and provide the appropriate 3D environment for ACI addition. MACI ${ }^{(}($Verigen, Leverkusen, Germany) comprises the use of viable autologous chondrocytes expanded ex vivo for several weeks until reach 10 million cells. Then, they are seeded onto a porcine-derived Type I/III collagen membrane (ACI-Maix). It is indicated for the repair of full-thickness cartilage defects of the knee (grade III and IV).

\section{Conclusions}

Cartilage related diseases are on the top list concerns of the World Health Organization, being the prevention of articular cartilage degeneration a major health matter for which there are few effective solutions. Accordingly, significant efforts are being developed worldwide in the fields of tissue engineering and regenerative medicine, but full cartilage restoration remains a paramount challenge. One of the major goals is to produce biological substitutes to restore, maintain or improve tissue function, using biocompatible and biodegradable support structures, i.e., scaffolds, in conjunction with human cells. By gathering tissue engineering and regenerative medicine, researchers have been interested in developing alternative approaches for restoring joint functionality. For instance, the creation of constructs with a structure and composition resembling native cartilage and yielding similar mechanical behaviour. Despite the evolution of implants for cartilage tissue repair, there are several requirements that still need to be fulfilled to obtain a successful hyaline cartilage formation.

\section{Acknowledgements}

This research was supported by the European Regional Development Fund (FEDER), through COMPETE2020 under the PT2020 program (POCI-01-0145-FEDER-023423), by the Portuguese Foundation for Science and Technology (UID/Multi/04044/2013) and Centro2020 PAMI ROTEIRO/0328/2013 ( $\mathrm{N}^{\mathrm{o}}$ 022158).

\section{References}

[1] K. Schrobback, T.J. Klein, R. Crawford, Z. Upton, J. Malda, D.I. Leavesley, Effects of oxygen and culture system on in vitro propagation and redifferentiation of osteoarthritic human articular chondrocytes, Cell and Tissue Research 347 (2012) 649-663.

[2] W.S. Toh, E.H. Lee , T. Cao, Potential of human embryonic stem cells in cartilage tissue engineering and regenerative medicine, Stem cell reviews 7 (2011) 544-559.

[3] S.J. Bryant, K.A. Davis-Arehart, N. Luo, R.K. Shoemaker, J.A. Arthur, K.S. Anseth, Synthesis and Characterization of Photopolymerized Multifunctional Hydrogels: Water-Soluble Poly(Vinyl Alcohol) and Chondroitin Sulfate Macromers for Chondrocyte Encapsulation, Macromolecules 37 (2004) 6726-6733. 
[4] B.H. Thomas, J. Craig Fryman, K. Liu , J. Mason, Hydrophilic-hydrophobic hydrogels for cartilage replacement, Journal of the Mechanical Behavior of Biomedical Materials 2 (2009) 588595.

[5] S. Janjanin, W.J. Li, M.T. Morgan, R.M. Shanti , R.S. Tuan, Mold-shaped, nanofiber scaffoldbased cartilage engineering using human mesenchymal stem cells and bioreactor, Journal of Surgical Research 149 (2008) 47-56.

[6] R.J. Williams , R.H. Brophy, Decision Making in Cartilage Repair Procedures, in: R.J. Williams (Eds.), Cartilage Repair Strategies, Humana Press Inc., Totowa, NJ, 2007, pp. 37-53.

[7] K.R. Flik, N. Verma, B.J. Cole , B.R. Bach, Articular Cartilage: Structure, Biology, and Function, in: R.J.W. C (Eds.), Cartilage Repair Strategies, Humana Press Inc., Totowa, NJ, 2007, pp.

[8] D.G. Jones , L. Peterson, Autologous Chondrocyte Implantation, in: R.J. Williams (Eds.), Cartilage Repair Strategies, Humana Press Inc., Totowa, NJ, 2007, pp.

[9] K. Schrobback, T.J. Klein, R. Crawford, Z. Upton, J. Malda, D.I. Leavesley, Effects of oxygen and culture system on in vitro propagation and redifferentiation of osteoarthritic human articular chondrocytes, Cell and Tissue Research 347 (2012) 649-663.

[10] L. Zhao, Chondrogenic differentiation of stem cells in human umbilical cord stroma with PGA and PLLA scaffolds, Journal of Biomedical Science and Engineering 03 (2010) 1041-1049.

[11] M.I. Baker, S.P. Walsh, Z. Schwartz, B.D. Boyan, A review of polyvinyl alcohol and its uses in cartilage and orthopedic applications, Journal of Biomedical Materials Research Part B: Applied Biomaterials 100 (2012) 1451-1457.

[12] C. Chang, Gelatin-chondroitin-hyaluronan tri-copolymer scaffold for cartilage tissue engineering, Biomaterials 24 (2003) 4853-4858.

[13] T.D. Sargeant, A.P. Desai, S. Banerjee, A. Agawu, J.B. Stopek, An in situ forming collagenPEG hydrogel for tissue regeneration, Acta Biomaterialia 8 (2012) 124-132.

[14] A.M. Bhosale , J.B. Richardson, Articular cartilage: structure, injuries and review of management, British Medical Bulletin 87 (2008) 77-95.

[15] H. Shin, B.D. Olsen , A. Khademhosseini, The mechanical properties and cytotoxicity of cellladen double-network hydrogels based on photocrosslinkable gelatin and gellan gum biomacromolecules, Biomaterials 33 (2012) 3143-3152.

[16] R.A. Muzzarelli, F. Greco, A. Busilacchi, V. Sollazzo, A. Gigante, Chitosan, hyaluronan and chondroitin sulfate in tissue engineering for cartilage regeneration: a review, Carbohydrate Polymers 89 (2012) 723-739.

[17] D.R. Jeffrey, I. Watt, Imaging hyaline cartilage, The British Journal of Radiology 76 (2003) 777-787.

[18] D. Bosnakovski, M. Mizuno, G. Kim, S. Takagi, M. Okumura, T. Fujinaga, Chondrogenic differentiation of bovine bone marrow mesenchymal stem cells (MSCs) in different hydrogels: influence of collagen type II extracellular matrix on MSC chondrogenesis, Biotechnology and Bioengineering 93 (2006) 1152-1163.

[19] J.T. Oliveira, T.C. Santos, L. Martins, R. Picciochi, A.P. Marques, A.G. Castro, N.M. Neves, J.F. Mano, R.L. Reis, Gellan Gum Injectable Hydrogels for Cartilage Tissue Engineering Applications: In Vitro Studies and Preliminary In Vivo Evaluation, TISSUE ENGINEERING: Part A 16 (2010) 343-353.

[20] J. Zhu, Bioactive modification of poly(ethylene glycol) hydrogels for tissue engineering, Biomaterials 31 (2010) 4639-4656. 
[21] M. Volesky, T. Charlton , J.T. Deland, Articular Cartilage Repair Strategies in the Ankle Joint, in: R.J.W. C (Eds.), Cartilage Repair Strategies, Humana Press Inc., Totowa, NJ, 2007, pp.

[22] G. Vunjak-Novakovic, M. Radisic, Cell Seeding of Polymer Scaffolds, in: A.P. Hollander, P.V. Hatton (Eds.), Methods in Molecular Biology, vol. 238: Biopolymer Methods in Tissue Engineering, Humana Press Inc., Totowa, NJ, 2004, pp.

[23] R.U. Kleemann, D. Krocker, A. Cedraro, J. Tuischer, G.N. Duda, Altered cartilage mechanics and histology in knee osteoarthritis: relation to clinical assessment (ICRS Grade), Osteoarthritis Cartilage 13 (2005) 958-963.

[24] R. Cancedda, B. Dozin, P. Giannoni, R. Quarto, Tissue engineering and cell therapy of cartilage and bone, Matrix Biology 22 (2003) 81-91.

[25] G. Bentley, The surgical treatment of chondromalacia patallae, The Journal of Bone and Joint Surgery 60-B (1978)

[26] R.J. Williams , G.G. Niederauer, Articular Cartilage Resurfacing Using Synthetic Resorbable Scaffolds, in: R.J. Williams (Eds.), Cartilage Repair Strategies, Humana Press Inc., Totowa, NJ, 2007, pp. 115-135.

[27] S.H.M. Söntjens, D.L. Nettles, M.A. Carnahan, L.A. Setton , M.W. Grinstaff, Biodendrimerbased hydrogel scaffolds for cartilage Tissue Repair, Biomacromolecules 7 (2006) 310-316.

[28] R. Pörtner, S. Nagel-Heyer, C. Goepfert, P. Adamietz , N.M. Meenen, Bioreactor design for tissue engineering, Journal of Bioscience and Bioengineering 100 (2005) 235-245.

[29] H. Chiang, C.-C. Jiang, Repair of Articular Cartilage Defects: Review and Perspectives, Journal of the Formosan Medical Association 108 (2009)

[30] T.D. Sargeant, A.P. Desai, S. Banerjee, A. Agawu, J.B. Stopek, An in situ forming collagenPEG hydrogel for tissue regeneration, Acta Biomaterialia 8 (2012) 124-132.

[31] L. Peterson, D. Menche, D. Grande, M. Pitman, Chondrocyte transplantation - an experimental model in rabbits, Journal of Orthopaedic Surgery and Research 9 (1984)

[32] M. Brittberg, A. Lindahl, A. Nilsson, C. Ohlsson, O. Isaksson, L. Peterson, Treatment of deep cartilage defects in the knee with autologous chondrocyte transplantation, The New England Journal of Medicine 331 (1994)

[33] G.N. Homminga, S.K. Bulstra, P.S.M. Bouwmeester , A.J.V.D. Linden, Perichondral grafting for Cartilge lesions of the knee, The Journal of Bone and Joint Surgery 72-B (1990)

[34] A. Madeira, C.L.d. Silva, F.d. Santos, E. Camafeita, J.M.S. Cabral , I. Sá-Correia, Human Mesenchymal Stem Cell Expression Program upon Extended Ex-Vivo Ciltuvation, as Revealed by 2-DE-Based quantitative proteomics, PLOS ONE 7 (2012)

[35] R. Kuroda, K. Ishida, T. Matsumoto, T. Akisue, H. Fujioka, K. Mizuno, H. Ohgushi, S. Wakitani , M. Kurosaka, Treatment of a full-thickness articular cartilage defect in the femoral condyle of an athlete with autologous bone-marrow stromal cells, Osteoarthritis Cartilage 15 (2007) 226-231.

[36] C. Kasemkijwattana, S. Hongeng, S. Kesprayura, V. Rungsinaporn, K. Chaipinyo , K. Chansiri, Autologous Bone Marrow Mesenchymal Stem Cells Implantation for Cartilage Defects: Two Cases Report, Journal of the Medical Association of Thailand 94 (2011) 395-400.

[37] K.S. Lee, S.H. Cha, H.W. Kang, J.Y. Song, K.W. Lee, K.B. Ko , H.T. Lee, Effects of serial passage on the characteristics and chondrogenic differentiation of canine umbilical cord matrix derived mesenchymal stem cells, Asian-Australasian Journal of Animal Sciences 26 (2013) 588-595.

[38] N.A. Martino, S.J. Reshkin, E. Ciani , M.E. Dell'Aquila, Calcium-sensing receptor-mediated osteogenic and early-stage neurogenic differentiation in umbilical cord matrix mesenchymal stem cells from a large animal model, PLoS One 9 (2014) e111533. 
[39] X. Zhang, M. Hirai, S. Cantero, R. Ciubotariu, L. Dobrila, A. Hirsh, K. Igura, H. Satoh, I. Yokomi, T. Nishimura, S. Yamaguchi, K. Yoshimura, P. Rubinstein, T.A. Takahashi, Isolation and characterization of mesenchymal stem cells from human umbilical cord blood: reevaluation of critical factors for successful isolation and high ability to proliferate and differentiate to chondrocytes as compared to mesenchymal stem cells from bone marrow and adipose tissue, Journal of Cellular Biochemistry 112 (2011) 1206-1218.

[40] L. Wu, X. Cai, S. Zhang, M. Karperien, Y. Lin, Regeneration of articular cartilage by adipose tissue derived mesenchymal stem cells: perspectives from stem cell biology and molecular medicine, Journal of Cellular Physiology 228 (2013) 938-944.

[41] N. Kohli, K.T. Wright, R.L. Sammons, L. Jeys, M. Snow , W.E.B. Johnson, An In Vitro Comparison of the Incorporation, Growth, and Chondrogenic Potential of Human Bone Marrow versus Adipose Tissue Mesenchymal Stem Cells in Clinically Relevant Cell Scaffolds Used for Cartilage Repair, Cartilage (2015)

[42] T. Olee, S.P. Grogan, M.K. Lotz, C.W. Colwell, Jr., D.D. D'Lima , E.Y. Snyder, Repair of cartilage defects in arthritic tissue with differentiated human embryonic stem cells, Tissue Engineering Part A 20 (2014) 683-692.

[43] A.M. Craft, N. Ahmed, J.S. Rockel, G.S. Baht, B.A. Alman, R.A. Kandel, A.E. Grigoriadis , G.M. Keller, Specification of chondrocytes and cartilage tissues from embryonic stem cells, Development 140 (2013) 2597-2610.

[44] R.A. Rezende, P.J. Bártolo, A. Mendes , R.M. Filho, Rheological behavior of alginate solutions for biomanufacturing, Journal of Applied Polymer Science 113 (2009) 3866-3871.

[45] P.A. Gunatillake , R. Adhikari, Biodegradable synthetic polymers for tissue engineering, European Cells and Materials 5 (2003) 1-16. 